\title{
Application of the Asymptotic Solution to EM Field Scattering Problem for Creation of Media with Prescribed Permeability
}

\author{
A. G. Ramm ${ }^{1}$, M. I. Andriychuk ${ }^{2}$ \\ ${ }^{1}$ Department of Mathematics \\ Kansas State University, Manhattan, KS 66506-2602, USA \\ ramm@math.ksu.edu \\ ${ }^{2}$ Institute for Applied Problems in Mechanics and Mathematics, NASU \\ Naukova St., 3B, 79060, Lviv, Ukraine \\ andr@iapmm.lviv.ua
}

October 18, 2013

\begin{abstract}
Scattering of electromagnetic (EM) waves by many small impedance particles (bodies), embedded in a homogeneous medium, is studied. Physical properties of the particles are described by their boundary impedances. The limiting equation is obtained for the effective EM field in the limiting medium, in the limit $a \rightarrow 0$, where $a$ is the characteristic size of a particle and the number $M(a)$ of the particles tends to infinity at a suitable rate. The proposed theory allows one to create a medium with a desirable spatially inhomogeneous permeability. The main new physical result is the explicit analytical formula for the permeability $\mu(x)$ of the limiting medium. The computational results confirm a possibility to create the media with various distributions of $\mu(x)$.
\end{abstract}

Keywords: EM Wave Scattering, Permeability, Modeling Results.

\section{Introduction}

A theory of electromagnetic (EM) wave scattering by many small impedance particles (bodies) embedded in a homogeneous medium with a constant permittivity $\varepsilon_{0}>0$, permeability $\mu_{0}>0$ and, possibly, constant conductivity $\sigma_{0} \geq 0$ is applied for creating a media with a prescribed permeability. The computational procedure for numerical solution of the scattering problem were developed in [1].

In [9] there is a mathematical analysis of the Maxwell's equations, and in [10] theory of the wave scattering by obstacles is developed. 
The small particles are embedded in a finite domain $D$. The medium, created by the embedding of the small particles, has new physical properties. In particular, it has a spatially inhomogeneous magnetic permeability $\mu(x)$, which can be controlled by the choice of the boundary impedances of the embedded small particles and their distribution density. An analytic formula for the permeability of the new medium is obtained (see Subsection 2.3).

Although the initial medium has a constant permeability $\mu_{0}$, the limiting medium, obtained as a result of embedding many small particles with prescribed boundary impedances, has a non-homogeneous permeability which is expressed analytically in terms of the distribution density of the small particles and their boundary impedances. Therefore, a new physical phenomenon is predicted theoretically, namely, appearance of a spatially inhomogeneous permeability as a result of embedding of many small particles whose physical properties are described by their boundary impedances.

We assume that in any sub-domain $\Delta$, the number $N(\Delta)$ of the embedded particles is given by the formula:

$$
\mathcal{N}(\Delta)=\frac{1}{a^{2-\kappa}} \int_{\Delta} N(x) d x[1+o(1)], \quad a \rightarrow 0,
$$

where $N(x) \geq 0$ is a continuous function, vanishing outside of the finite domain $D$ in which small particles (bodies) are distributed, $\kappa \in(0,1)$ is a number that one can choose as one wishes, and the boundary impedances of the small particles are defined by the formula:

$$
\zeta_{m}=\frac{h\left(x_{m}\right)}{a^{\kappa}}, \quad x_{m} \in D_{m}, \quad \operatorname{Re} h(x) \geq 0,
$$

where $x_{m}$ is a point inside $m$-th particle, and $h(x)$ is a continuous function vanishing outside $D$ which satisfies only the physical restriction $\operatorname{Reh}(x) \geq 0$. The function $h$, used in our numerical examples satisfies this restriction. The impedance boundary condition on the surface $S_{m}$ of the $m$-th particle $D_{m}$ is $E^{t}=\zeta_{m}\left[H^{t}, N\right]$, where $E^{t}\left(H^{t}\right)$ is the tangential component of $E(H)$ on $S_{m}$, and $N$ is the unit normal to $S_{m}$, pointing out of $D_{m}$.

Since one can choose the functions $N(x)$ and $h(x)$, one can create a desired magnetic permeability in $D$. This is a novel idea proposed originally in [14], [15]. It has led to a recipe for creating materials with a desired refraction coefficient in [19], [20]. [21].

Materials with negative permittivity and permeability are of interest in applications, see [11], [23], [24]).

We also derive an analytic formula for the refraction coefficient of the medium in $D$ created by the embedding of many small particles. An equation for the effective EM field in the limiting medium is derived. This medium is created when the size $a$ of small particles tends to zero while the total number $M=M(a)$ of the particles tends to infinity at a suitable rate.

The refraction coefficient $n^{2}(x)$ in the limiting medium is spatially inhomogeneous. 
The proposed theory may be viewed as a "homogenization theory", but it differs from the usual homogenization theory (see, e.g., [3], [10], [5], and references therein) in several respects: we do not assume any periodic structure in the distribution of small bodies, our problem does not have a discrete spectrum, our operators are non-selfadjoint, to mention some of the differences. The ideas, methods, and techniques are also quite different from the usual methods. These ideas are similar to the ideas developed in papers [15], [16], where scalar wave scattering by small bodies was studied. More on this one can find in the forthcoming monograph [22].

However, the scattering of EM waves brought new technical difficulties which are resolved in this paper. The difficulties come from the vectorial nature of the boundary conditions. Our approach is valid for small particles of arbitrary shape, but for simplicity we assume that the small bodies are balls of radius $a$ $[18]$.

\section{EM Wave Scattering by Many Small Particles}

\subsection{Statement of Problem}

It is assumed that many small bodies $D_{m}, 1 \leq m \leq M$, are embedded in a homogeneous medium with constant parameters $\varepsilon_{0}, \mu_{0}$. Let $k^{2}=\omega^{2} \varepsilon_{0} \mu_{0}$, where $\omega$ is the frequency. The arguments remain valid if one assumes that the medium has a constant conductivity $\sigma_{0}>0$. In this case $\varepsilon_{0}$ is replaced by $\varepsilon_{0}+i \frac{\sigma_{0}}{\omega}$. Denote by $[E, H]=E \times H$ the cross product of two vectors, and by $(E, H)=E \cdot H$ the dot product of two vectors.

EM wave scattering problem consists of finding vectors $E$ and $H$ satisfying the Maxwell equations:

$$
\nabla \times E=i \omega \mu_{0} H, \quad \nabla \times H=-i \omega \varepsilon_{0} E
$$

in $D:=\mathbb{R}^{3} \backslash \bigcup_{m=1}^{M} D_{m}$, the impedance boundary conditions:

$$
[N,[E, N]]=\zeta_{m}[N, H]
$$

on $S_{m}, 1 \leq m \leq M$, and the radiation conditions:

$$
E=E_{0}+v_{E}, \quad H=H_{0}+v_{H},
$$

where $\zeta_{m}$ is the impedance, $N$ is the unit normal to $S_{m}$ pointing out of $D_{m}$, $E_{0}, H_{0}$ are the incident fields satisfying equations (3) in all of $\mathbb{R}^{3}$. The usual form of impedance boundary condition is $E^{t}=\zeta\left[N, H^{t}\right]$, where $E^{t}:=[N,[E, N]]$ is the tangential component of $E$ on $S$. Thus, $\left[N, H^{t}\right]=[N,[N,[H, N]]]=[N, H]$, because $[N,[H, N]]=H-N H \cdot N$, and $[N, N]=0$. This justifies the impedance boundary condition (4) we use. The impedance boundary condition is widely applicable in physics. We assume in this paper that the impedance $\zeta$ is a constant, $\operatorname{Im} \zeta \geq 0$. In the literature (see, for example, [9]) one may see the 
impedance boundary condition with $\left[H^{t}, N\right]$ in place of our $\left[N, H^{t}\right]$. This is due to the fact that $N$ in [9] is the unit normal to $S$ pointing into $D$, while we use $N$ pointing out of $D$.

One often assumes that the incident wave is a plane wave, i.e., $E_{0}=\beta e^{i k \alpha \cdot x}, \beta$ is a constant vector, $\alpha \in S^{2}$ is a unit vector, $S^{2}$ is the unit sphere in $\mathbb{R}^{3}, \alpha \cdot \beta=0$, $v_{E}$ and $v_{H}$ satisfy the radiation condition:

$$
r\left(\frac{\partial v}{\partial r}-i k v\right)=o(1)
$$

Impedance $\zeta_{m}$ is assumed to be a constant, $\operatorname{Re} \zeta_{m} \geq 0$, so that

$$
\operatorname{Re}\left(\zeta_{m} E^{t}, E^{t}\right) \geq 0 \forall E^{t} \in T_{m},
$$

where $T_{m}$ is the set of all tangential to $S_{m}$ continuous vector fields such that $\operatorname{Div} E^{t}=0$, where Div is the surface divergence, and $E^{t}$ is the tangential component of $E$. Smallness of $D_{m}$ means that $k a \ll 1$, where

$$
a=0.5 \max _{1 \leq m \leq M} \operatorname{diam} D_{m}
$$

Our definition of $E^{t}$ is:

$$
E^{t}=E-N(E, N)=[N,[E, N]]
$$

This definition differs from the one used often in the literature, namely, from the definition $E^{t}=[N, E]$. Since

$$
H=\frac{\nabla \times E}{i \omega \mu_{0}},
$$

one gets

$$
\nabla \times \nabla \times E=k^{2} E \text { in } D,
$$

and the impedance boundary condition is

$$
[N,[E, N]]=\frac{\zeta_{m}}{i \omega \mu_{0}}[N, \nabla \times E]
$$

on $S_{m}, 1 \leq m \leq M$.

Thus, we have reduced problem (3)-(5) to finding one vector $E(x)$ satisfying the impedance boundary condition (11). If $E(x)$ is found, then $H=\frac{\nabla E}{i \omega \mu_{0}}$.

\subsection{Finding the Solution}

Let us look for $E$ of the form

$$
\begin{aligned}
& E=E_{0}+\sum_{m=1}^{M} \nabla \times \int_{S_{m}} g(x, t) \sigma_{m}(t) d t \\
& g(x, y)=\frac{e^{i k|x-y|}}{4 \pi|x-y|}
\end{aligned}
$$


where $t \in S_{m}$ and $d t$ is an element of the area of $S_{m}, \quad \sigma_{m}(t) \in T_{m}$. This $E$ for any continuous $\sigma_{m}(t)$ solves equation (10) in $D$ because $E_{0}$ solves (10).

Define the effective field $E_{e}(x)=E_{e}^{m}(x)=E_{e}^{(m)}(x, a)$, acting on the $m$-th body $D_{m}$, by the formula:

$$
E_{e}(x)=E(x)-\nabla \times \int_{S_{m}} g(x, t) \sigma_{m}(t) d t:=E_{e}^{(m)}(x),
$$

where it is assumed that $x$ is in a neighborhood of $S_{m}$, but $E_{e}(x)$ is defined for all $x \in \mathbb{R}^{3}$. Let $x_{m} \in D_{m}$ be a point inside $D_{m}$, and $d=d(a)$ be the distance between two neighboring small bodies. Let us assume that

$$
\lim _{a \rightarrow 0} \frac{a}{d(a)}=0, \quad \lim _{a \rightarrow 0} d(a)=0 .
$$

It is proved in [15] that $E_{e}(x, a)$ tends to a limit $E_{e}(x)$ as $a \rightarrow 0$, and $E_{e}(x)$ is a twice continuously differentiable function.

Let us assume that in any sub-domain $\Delta$, the number $\mathcal{N}(\Delta)$ of the embedded bodies $D_{m}$ is given by formula (1), and boundary impedances $\zeta_{m}$ of small particles are defined by formula (2).

Let us write (12) as

$$
\begin{aligned}
& E(x)=E_{0}(x)+\sum_{m=1}^{M}\left[\nabla_{x} g\left(x, x_{m}\right), Q_{m}\right]+ \\
& \sum_{m=1}^{M} \nabla \times \int_{S_{m}}\left(g(x, t)-g\left(x, x_{m}\right)\right) \sigma_{m}(t) d t,
\end{aligned}
$$

where

$$
Q_{m}:=\int_{S_{m}} \sigma_{m}(t) d t
$$

Since $\sigma_{m}=O\left(a^{-\kappa}\right)$, one has $Q_{m}=O\left(a^{2-\kappa}\right)$. One wants to prove that the second sum in (15) is negligible compared with the first one. This proof is based on several estimates. These estimates show that one may neglect the second sum in (15), see [18], and write

$$
E_{e}(x)=E_{0}(x)+\sum_{m=1}^{M}\left[\nabla_{x} g\left(x, x_{m}\right), Q_{m}\right]
$$

with an error that tends to zero under our assumptions as $a \rightarrow 0$, and when $\left|x-x_{j}\right| \sim a$ then the term with $m=j$ in the sum (17) should be dropped according to the definition of the effective field. We will show that the limit of the effective field, as $a \rightarrow 0$ does exist and solves equation (21), see below.

As $a \rightarrow 0$, the sum in (17) converges to the integral

$$
E(x)=E_{0}(x)+\nabla \times \int_{D} g(x, y) N(y) Q(y) d y,
$$


where $Q(y)$ is the function uniquely defined by the formula

$$
Q_{m}=Q\left(x_{m}\right) a^{2-\kappa}
$$

where $Q(y)$ is a continuous function in $D$. This function $Q(y)$ is defined uniquely, because, as $a \rightarrow 0$ the set of points $\left\{x_{m}\right\}_{m=1}^{M}$ becomes dense in $D$. The physical meaning of vector $E(x)$ in equation (18) is clear: this vector is the limit of the effective field $E_{e}(x)$ as $a \rightarrow 0$, and $N(x)$ is the function from equation (1).

The function $Q(y)$ can be expressed in terms of $E$ :

$$
Q(y)=\frac{8 \pi i}{3 \omega \mu_{0}} h(y)(\nabla \times E)(y) .
$$

The factor $\frac{8 \pi}{3}$ appears if $D_{m}$ are balls. Otherwise a tensorial factor $c_{m}$, depending on the shape of $S_{m}$, should be used in place of $\frac{8 \pi}{3}$.

From equations (18) and (20) one obtains

$$
E(x)=E_{0}(x)+\frac{8 \pi i}{3 \omega \mu_{0}} \nabla \times \int_{D} g(x, y) h(y) N(y) \nabla \times E(y) d y .
$$

\subsection{Explicit Formula for Permeability of Resulting Medium}

Let us derive main physical conclusion from equation (21). Applying the operator $\nabla \times \nabla \times$ to $(21)$ yields

$$
\begin{aligned}
& \nabla \times \nabla \times E=k^{2} E_{0}(x)+\frac{8 \pi i}{3 \omega \mu_{0}} \nabla \times(\text { grad div }- \\
& \left.\nabla^{2}\right) \int_{D} g(x, y) h(y) N(y) \nabla \times E(y) d y \\
& =k^{2} E(x)+\frac{8 \pi i}{3 \omega \mu_{0}} h(x) N(x) \nabla \times \nabla \times E+\frac{8 \pi i}{3 \omega \mu_{0}}[\nabla(h(x) N(x)), \nabla \times E(x)] .
\end{aligned}
$$

Here we have used the formula $\nabla \times \operatorname{grad}=0$ and the equation

$$
-\nabla^{2} g(x, y)=k^{2} g(x, y)+\delta(x-y),
$$

and took into account that $h(x)$ is a scalar function by the assumption.

It follows from (22) that

$$
\nabla \times \nabla \times E=K^{2}(x) E+\frac{\frac{8 \pi i}{3 \omega \mu_{0}}}{1-\frac{8 \pi i}{3 \omega \mu_{0}} h(x) N(x)}[\nabla(h(x) N(x)), \nabla \times E(x)],
$$

where

$$
K^{2}(x)=\frac{k^{2}}{1-\frac{8 \pi i}{3 \omega \mu_{0}} h(x) N(x)}, k^{2}=\omega^{2} \varepsilon_{0} \mu_{0} .
$$

If one uses the equation 


$$
\nabla \times E=i \omega \mu(x) H, \quad \nabla \times H=-i \omega \varepsilon(x) E
$$

then

$$
\nabla \times \nabla \times E=\omega^{2} \varepsilon(x) \mu(x) E+\left[\frac{\nabla \mu(x)}{\mu(x)}, \nabla \times E\right] .
$$

Comparing this equation with (24), one can identify the last term in (24) as coming from a variable permeability $\mu(x)$. This $\mu(x)$ appears in the limiting medium due to the boundary currents on the surfaces $S_{m}, 1 \leq m \leq M$. These currents appear because of the impedance boundary conditions (11).

Let us identify the permeability $\mu(x)$. Denote

$$
\Psi(x):=1-\frac{8 \pi i}{3 \omega \mu_{0}} h(x) N(x) .
$$

Let $\varepsilon(x)=\varepsilon_{0}, \varepsilon_{0}=$ const, and define

$$
\mu(x):=\frac{\mu_{0}}{\Psi(x)} .
$$

Then $K^{2}=\omega^{2} \varepsilon_{0} \mu(x)$, and

$$
\frac{\nabla \mu(x)}{\mu(x)}=\frac{\nabla \Psi(x)}{\Psi(x)} .
$$

Consequently, formula (24) has a clear physical meaning: the electromagnetic properties of the limiting medium are described by the variable permeability:

$$
\mu(x)=\frac{\mu_{0}}{\Psi(x)}=\frac{\mu_{0}}{1-\frac{8 \pi i}{3 \omega \mu_{0}} h(x) N(x)},
$$

and the limiting medium is described by the new refraction coefficient $K^{2}=$ $\omega^{2} \varepsilon_{0} \mu(x)$.

\section{Derivation of a Linear Algebraic System for Vectors $P_{m}$}

In this Section a numerical method is developed for solving many-body wave scattering problem when the scatterers (bodies) are small in comparison with the wavelength. The method consists of a derivation of a linear algebraic system for finding vectors

$$
P_{m}=(\nabla \times E)\left(x_{m}\right), \quad 1 \leq m \leq M .
$$

If $P_{m}$ are found, then by formulas (20) and (19) one finds

$$
Q_{m}=\frac{8 \pi i}{3 \omega \mu_{0}} a^{2-\kappa} h\left(x_{m}\right) P_{m}
$$


and, by formula (17), the field $E(x)$.

Let us derive linear algebraic system for finding $P_{m}$. Apply the operator $\nabla \times$ to equation (21), let $x=x_{j}, 1 \leq j \leq M$, and replace $\sum_{m=1}^{M}$ by the sum $\sum_{m \neq j, m=1}^{M}$.

Then one obtains

$$
\begin{aligned}
& P_{j}=P_{0 j}+\frac{8 \pi i}{3 \omega \mu_{0}} a^{2-\kappa} \sum_{m \neq j, m=1}^{M}\left\{k^{2} g\left(x, x_{m}\right) h\left(x_{m}\right) P_{m}+\right. \\
& \left.h\left(x_{m}\right)\left(P_{m}, \nabla\right) \nabla g\left(x, x_{m}\right)\right\}\left.\right|_{x=x_{j}}
\end{aligned}
$$

where $1 \leq j \leq M$, and

$$
P_{0 j}:=\left(\nabla \times E_{0}\right)\left(x_{j}\right), \quad P_{j}=(\nabla \times E)\left(x_{j}\right), \quad 1 \leq j \leq M .
$$

Equation (32) is a linear algebraic system for finding $P_{m}$.

\section{$4 \quad$ Numerical Results}

The numerical calculations demonstrate the dependence of convergence of the iterative process for solving LAS (34)-(36) on the parameters $a, d$, and $M$. This allows one to establish the limits of the asymptotic approach depending on the parameters of problem and to investigate the dependence of the created permeability on $a$ and $M$.

Part of the numerical results justifies the assumptions $\operatorname{Div} E_{t}=0$ and $\operatorname{Div} \sigma_{m}=$ 0 , which can be used for obtaining the asymptotic solution (17).

The numerical examples demonstrate a possibility to create media with piecewise-constant permeability.

\subsection{Checking the Applicability of Asymptotic Approach}

The numerical experiments were carried out for checking the convergence of solution to LAS (32). It contains the unknown values of $P_{j}$ on the left-hand part, and on the right-hand part in the terms $\left(P_{m}, \nabla\right) \nabla g\left(x, x_{m}\right)$. The detailed form of LAS (32) can be given by the formulas:

$$
\begin{aligned}
& P_{j x}=P_{0 x}+\frac{8 \pi i}{3 \omega \mu_{0}} a^{2-\kappa} \sum_{m \neq j, m=1}^{M} h\left(x_{m}\right)\left[-P_{m x} \times\right. \\
& \left.\left(\frac{\partial^{2}}{\partial y^{2}}+\frac{\partial^{2}}{\partial z^{2}}\right) g\left(x, x_{m}\right)+P_{m y} \frac{\partial^{2}}{\partial x \partial y} g\left(x, x_{m}\right)+P_{m z} \frac{\partial^{2}}{\partial x \partial z} g\left(x, x_{m}\right)\right], \\
& P_{j y}=P_{0 y}+\frac{8 \pi i}{3 \omega \mu_{0}} a^{2-\kappa} \sum_{m \neq j, m=1}^{M} h\left(x_{m}\right)\left[-P_{m y} \times\right. \\
& \left.\left(\frac{\partial^{2}}{\partial x^{2}}+\frac{\partial^{2}}{\partial z^{2}}\right) g\left(x, x_{m}\right)+P_{m x} \frac{\partial^{2}}{\partial x \partial y} g\left(x, x_{m}\right)+P_{m z} \frac{\partial^{2}}{\partial y \partial z} g\left(x, x_{m}\right)\right],
\end{aligned}
$$




$$
\begin{aligned}
& P_{j z}=P_{0 z}+\frac{8 \pi i}{3 \omega \mu_{0}} a^{2-\kappa} \sum_{m \neq j, m=1}^{M} h\left(x_{m}\right)\left[-P_{m z} \times\right. \\
& \left.\left(\frac{\partial^{2}}{\partial x^{2}}+\frac{\partial^{2}}{\partial y^{2}}\right) g\left(x, x_{m}\right)+P_{m x} \frac{\partial^{2}}{\partial x \partial z} g\left(x, x_{m}\right)+P_{m y} \frac{\partial^{2}}{\partial y \partial z} g\left(x, x_{m}\right)\right],
\end{aligned}
$$

where $P_{j x}, P_{j y}$, and $P_{j z}$ are the $x-, y$-, and $z$-components of vectors $P_{j}$, respectively. One can see from (34)-(36) that for determination of $P_{j x}$ component it is necessary to know the remaining components $P_{j y}$ and $P_{j z}$. The following iterative process is proposed for solving LAS (34)-(36):

Step 1. Let $n=0$ is number of iteration.

Step 2. The initial approximations $P_{m y n}$ and $P_{m z n}$ are prescribed (or given if $n \neq 0$ ), and vector $P_{j x n+1}$ is determined by solving (34).

Step 3. Having $P_{m x n+1}$ and $P_{m z n}$, we determine $P_{m y n+1}$ from (35).

Step 4 . We determine $P_{m z n+1}$ with know $P_{m x n+1}, P_{m y n+1}$ solving LAS (36).

Step 5. If the inequalities

$$
\begin{gathered}
\left|P_{m x_{n+1}}-P_{m x_{n}}\right|<\varepsilon, \\
\left|P_{m y_{n+1}}-P_{m y_{n}}\right|<\varepsilon, \\
\left|P_{m z_{n+1}}-P_{m z_{n}}\right|<\varepsilon,
\end{gathered}
$$

are satisfied (there $\varepsilon$ is the given accuracy of calculations), we finish the iterative procedure. Otherwise, we return to Step 2, by setting $n=n+1$.

It is obvious that the inequalities are not checked in the first iteration, because $P_{m x 0}$ is not given.

It was established that convergence of the above iterative process depends on the ratio $d$ to $a$ at the fixed value of $M$. The calculations for a series of $d$ show that the value $d / a \approx 10$ is the lowest one, that provides the convergence of the iterative method used. The iterative procedure for solving LAS (34)-(36) for $d / a<10$ becomes unstable and does not converge if $d / a$ increase, but there are special cases when the value of the threshold is smaller.

In Fig. 1, the rate of convergence, depending on the radius $a$ of particle at the fixed number of particles in $D$ and the distance between them, is shown for $P_{m x}$ and $P_{m y}$ components. The relative error of the solution

$$
R E=\frac{\left\|S_{n+1}-S_{n}\right\|}{\left\|S_{n+1}\right\|}
$$

is put along the $y$ axis, and number of iteration $n$ is put along the $x$ axis. Here $S_{n}:=\left\{P_{m x n}, P_{m y n}, P_{m z n}\right\}$, and we use the sign $\|\cdot\|$ of the norm because $P_{m x n}$, $P_{m y n}$, and $P_{m z n}$ are the matrices. The number of particles is equal to $10^{3}$, and the distance between them is equal to 0.5. The linear distances $(a$ and $d$ ) are measured in $\mathrm{cm}\left(1 \times 10^{-2} \mathrm{~m}\right)$. The maximal error is observed in the first iteration. This error is equal to 0.075 and 0.004 for $a=0.08$ and $a=0.01$, 


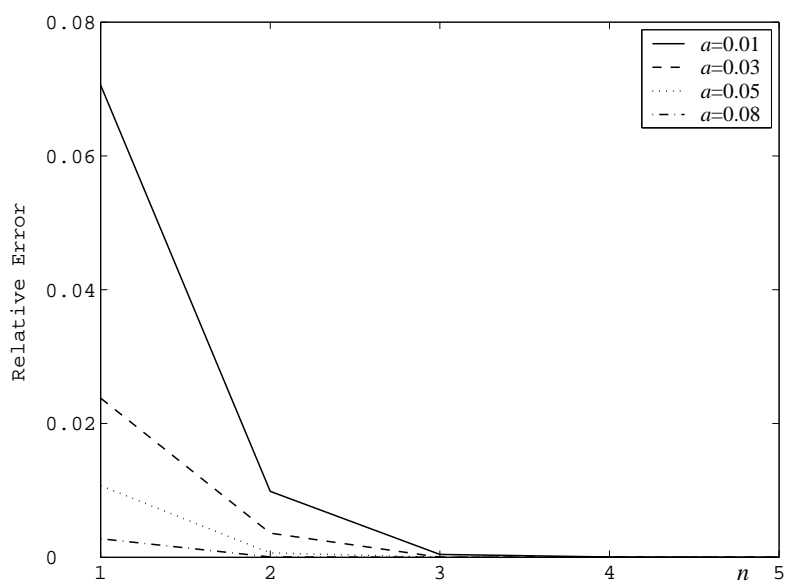

Figure 1: The relative error versus number $n$ of iteration for $P_{m x}$ component

respectively. The error decreases quickly and in the third iteration it is equal to $2 \times 10^{-4}$ and $5.6 \times 10^{-9}$ for the above values of $a$.

The relative error for the $P_{m y}$ component behaves similarly, although their values are slightly smaller: they are equal to 0.066 and 0.003 for $a=0.08$ and $a=0.01$, respectively.

The properties of the resulting medium depends on the radius $a$ of the particles when $M$ and $d$ are fixed. In Table 1 , the permeability $\mu$, wave number $k$, and maximal value of amplitude $E_{x}$ of the scattered field are given for the values of $a$, considered in the previous example. The values of $\mu$ are normalized on the quantity $\mu_{0}=4 \pi \times 10^{-7} \mathrm{H} \times \mathrm{m} \approx 1.256 \times 10^{-6} \mathrm{H} \times \mathrm{m}$. At such a normalization, $\mu_{0}=1$. One can see that value of $\mu$ is changed by $14 \%$ for $a=0.01$ and by $58 \%$ for $a=0.08$. The maximal value of the component $E_{x}$ of the scattered field increased almost tenfold when $a$ is changed from 0.01 to 0.08 .

The iterative process becomes unstable as $a$ grows, and this process does not converges if $d / a<6$ at the considered values of $M$ and $d$.

Table 1. The properties of resulting domain $D$ at various $a$.

$\begin{array}{lllll} & a=0.01 & a=0.03 & a=0.05 & a=0.08 \\ \mu & 0.8641 & 0.6616 & 0.5345 & 0.4196 \\ k & 0.0930 & 0.0814 & 0.0731 & 0.0646 \\ \max \left(E_{x}\right) & 1.1569 & 1.5308 & 1.94191 & 2.61896\end{array}$

In Fig. 3, the values of the permeability $\mu$ are calculated by formula 29 and their dependence on $a$ is shown. The value of $k=10 \mathrm{~m}^{-1}$ corresponds to the frequency $\omega=30 \mathrm{MHz}$, while the radius $a$ of the particles changes between the limits $1 \times 10^{-4} \mathrm{~m} \div 9 \times 10^{-4} \mathrm{~m}, h(x)=10 i$.

One can see that the values of $\mu$ for larger $d$ are closer to the initial permeability $\mu_{0}=1$ of the medium without embedded particles. This is clear, 


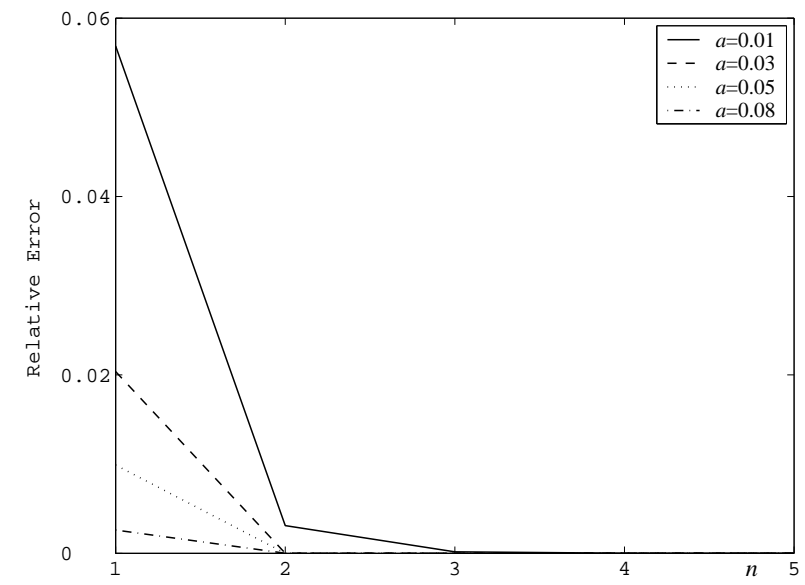

Figure 2: The relative error versus number $n$ of iteration for $P_{m y}$ component

because as the values of $d$ increase, the physical properties of $D$ become closer to properties of the initial medium. The results shown for the values of $d / a$ which assure the convergence of the proposed iterative process.

The results, presented in Fig. 4, demonstrate the change of $\mu$ for various $d$ at the optimal physical parameters of $D$ which guarantee the convergence of the iterative process for solving LAS (34)-(36). One can see that for the smaller $d$ the permeability changes considerably. For example, at $d=0.3$ and $a=0.01$ the values of $\mu$ deviate from $\mu_{0}=1.0$ by $40 \%$. This deviation decreases as $d$ grows, and it does not exceed $5 \%$ at $d=0.9$ for the considered range of $a$. The value of the function $h(x)$ influences the behavior of $\mu$. The results shown in Fig. 5 demonstrate that the values of $\mu$ corresponding to the small $|\operatorname{Im} h(x)|$ are closer to $\mu_{0}=1$, and the difference between $\mu$ and $\mu_{0}$ grows if $|\operatorname{Im} h(x)|$ increases. The convergence of the solution to LAS (34)-(36) depends on the values of $|\operatorname{Im} h(x)|$. At the small values of $d$ the convergence holds for small values of $|\operatorname{Im}(h)|$ and the convergence region increases as the value of $d$ increases.

The other characteristics of the field change if the radius $a$ and the function $h(x)$ change. The amplitude of the scattered field $E$ is one of such characteristics. In Fig. 6, the dependence of the maximum of the amplitude $E_{x}$ of the scattered field on the radius $a$ of the particles is shown. This amplitude grows if $a$ increases. The increase of the amplitude is similar for various $d$ and the amplitude changes almost linearly as the radius $a$ of the particles grows. The amplitude increases at $d=0.9$ more than ten times when $k a$ increases from 0.01 up to 0.09. Referring to Fig. 3, one sees that the amplitude grows as a grows and the values of $\mu$ deviate from the initial value $\mu_{0}$. 


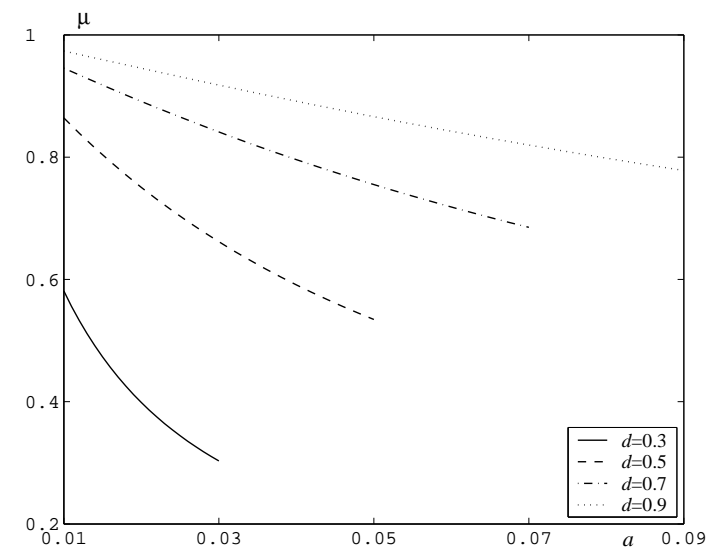

Figure 3: The values of permeability $\mu$ versus the radius of particle $a$

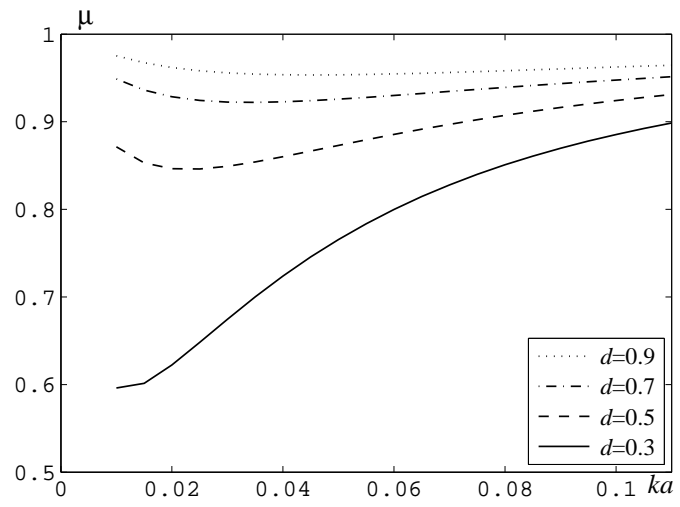

Figure 4: The values of permeability $\mu$ at the optimal parameters of $D$ 


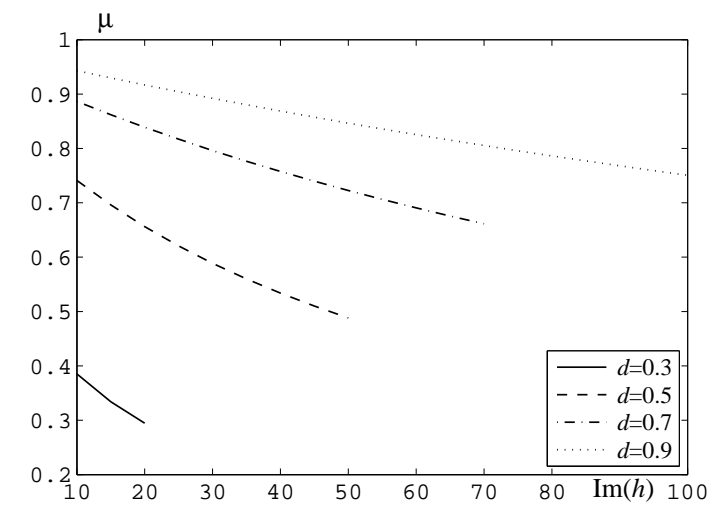

Figure 5: The values of permeability $\mu$ versus the value of $\operatorname{Im} h(x)$

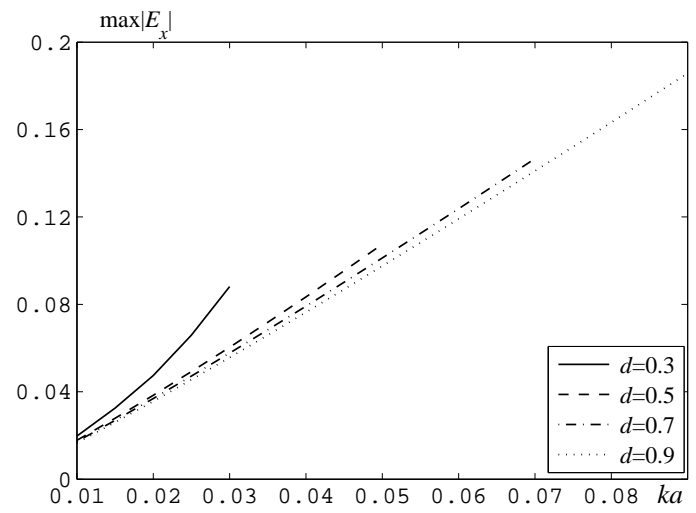

Figure 6: Dependence of $\max \left|E_{x}\right|$ on the radius $a$ of particle 


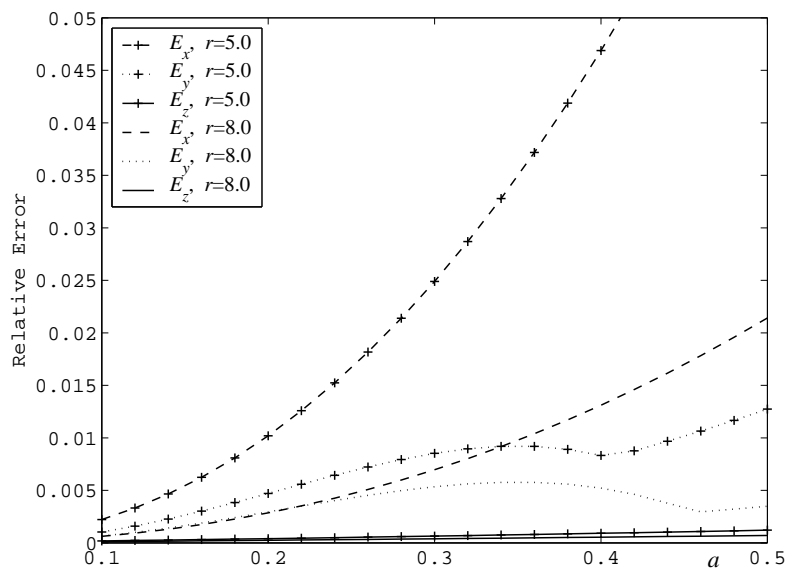

Figure 7: Relative error of $E_{x}, E_{y}$, and $E_{z}$ components for various distances $r$ to observation point, $h(x)=10 i$

\subsection{Exactness of Asymptotic Formula (17)}

It is important from the practical point of view to know how accurate is asymptotic formula (17). To answer on this question we carry out a series of computations related to the comparison of asymptotic formula (17) with the solution of EM wave scattering problem by Mie series, obtained in [2]. We consider here the particular case $M=1$, because the EM wave scattering problem for one particle was considered there. Solution (52) in the form of Mie series is considered as the benchmark one. We use the formulas of transformation of the electrical field's spherical components into cartesian ones [7] because solution (52) is given in the spherical coordinates. The relative error is defined as $\left\|E_{52}-E_{17}\right\| /\left\|E_{52}\right\|$, where $\|\cdot\|$ is the sup norm, $E=\left(E_{x}, E_{y}, E_{z}\right)$. The subscript 52 indicates the solution obtained by Mie series (52) in [2], and subscript 17 indicates the solution obtained by asymptotic formula (17). The calculations are performed for series of radius $a$ of particle and various functions $h(x)$.

In Fig. 7 , the relative error of the $E_{x}, E_{y}$, and $E_{z}$ components depending on the radius $a$ of particles is shown for several distances $r$ from the center of particle to the observation point, $k=0.1, h(x)=10 i$. One can observe that the relative error is very sensitive to the radius $a$ of particle. The maximal value of error is attained for $E_{x}$ component at $a=0.5$ and it is equal to $7.6 \%$. The values of relative error for $E_{y}$ and $E_{z}$ components are $1.2 \%$ and $0.1 \%$, respectively. The minimal values of error for all components are reached at $a=0.1$ and they are equal to $0.2 \%, 0.1 \%$, and $0.02 \%$ for $E_{x}, E_{y}$, and $E_{z}$, respectively. The relative error diminishes quickly as distance $r$ grows. So, the maximal values of error for $r=8.0$ are observed at $a=0.5$ and they are equal to $2.1 \%, 0.3 \%$, and $0.07 \%$ for for $E_{x}, E_{y}$, and $E_{z}$ components. This error does not exceed $0.06 \%$ at $a=0.1$. The numerical calculations show that the relative error depends on the function 


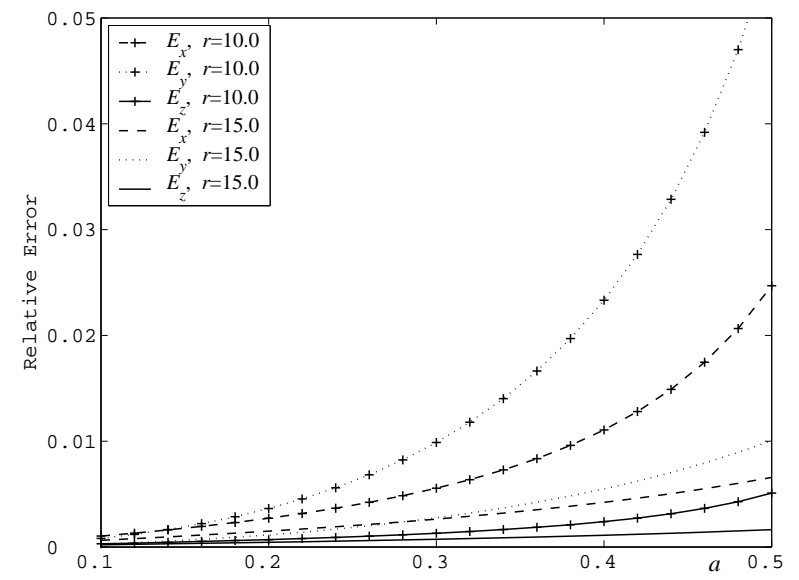

Figure 8: Relative error of $E_{x}, E_{y}$, and $E_{z}$ components for various distances $r$ to observation point, $h(x)=100 i$

$h(x)$ influencing the value of surface impedance $\zeta$ considerably. In Fig. 8 , the values of relative error are shown for $h(x)=100 i$. It turned out that relative error increases too much. For example, its value for $E_{x}$ component at $r=8.0$ is equal to $25.1 \%$, the values of this error for $E_{y}$ and $E_{z}$ components are a little smaller. In order to attain the relative error of the same order as in the previous example, it is necessary to increase the distance $r$ approximately twice. So, the relative error of $E_{y}$ component at $r=10.0$ for $a=0.5$ is equal to $5.7 \%$; the minimal value of error at these $r$ and $a$ is reached for $E_{z}$ component and it is equal to $0.5 \%$. The maximal value of error at $r=15.0$ is equal to $1.0 \%$ and $0.02 \%$ for a $=0.5$ and $a=0.1$, respectively, and these values are attained for $E_{y}$ component. The relative error for $E_{x}$ and $E_{z}$ components is smaller.

The obtained computational results testify the high accuracy of asymptotic formula (17) for the case of one particle. The relative error of (17) depends on the radius $a$ of particle, distance $r$ to point of observation (point in far zone), and function $h(x)$ influencing the value of surface impedance $\zeta$.

\subsection{Justification of Assumption $\operatorname{Div} E_{t}=0$}

Is it possible to use the assumption $\operatorname{Div} E_{t}=0$ on the surfaces of the particles? This assumption is not justified theoretically. Therefore, the numerical justification of this assumption is of interest. We carry out a series of calculations which show that the equality $\operatorname{Div} E_{t}=0$ is satisfied with high accuracy. The derivatives $\frac{\partial E_{x}}{\partial x}, \frac{\partial E_{y}}{\partial y}$, and $\frac{\partial E_{z}}{\partial z}$ are replaced by the corresponding finite differences in the process of the numerical calculations of $\operatorname{Div} E_{t}$.

First, the investigation of the dependence of the value of $\operatorname{Div} E_{t}$ on the radius $a$ of particles at a fixed distance $d$ between them is done. The calculations are 


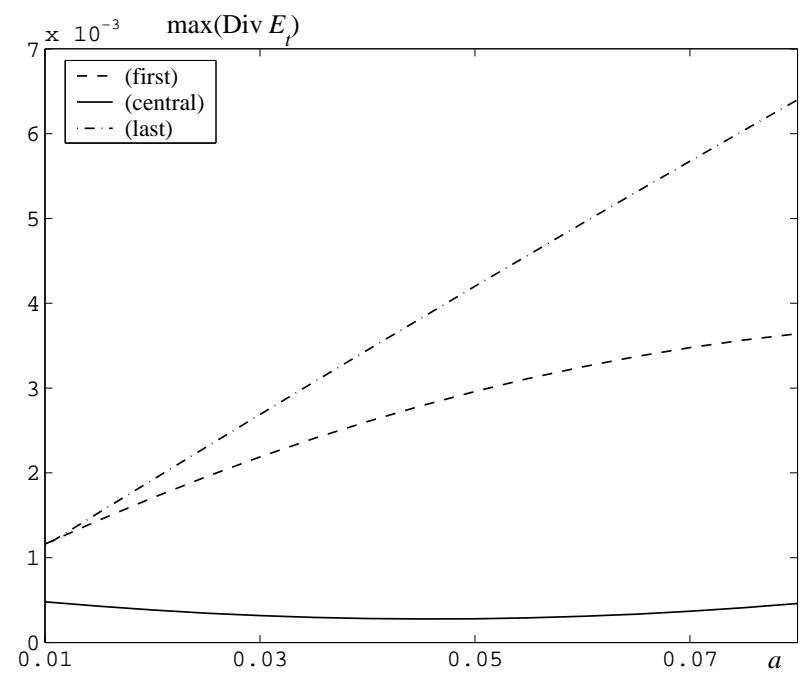

Figure 9: Maximal value of $\operatorname{Div} E_{t}$ versus radius $a$ of particle

carried out for $M=10^{3}, d=0.5$, and $k=0.1$. In Fig. 9, the maximal values of Div $E_{t}$ on the surface of the first, central, and last particles are shown. The numbering of particles starts from the left upper side of $D$ and continues to its right lower side. One can see that the maximal value of Div $E_{t}$ does not exceed $6.4 \times 10^{-3}, 3.7 \times 10^{-3}$, and $0.45 \times 10^{-3}$ for the first, central, and last particles, respectively. In Fig. 10, the minimal values of the $E_{x}$ and $E_{z}$ components on the surface of the particles are shown. In this case, the values $E_{x}$ and $E_{y}$ practically coincide. Comparing the results in both Figures, one concludes that the values of $\operatorname{Div} E_{t}$ does not exceed $2 \%$ in comparison with the corresponding values of $E$ components. So, the relation $\max \left(\operatorname{Div} E_{t}\right) / \min \left(E_{x, y, z}\right)$ equals to $1.4 \times 10^{-2}, 0.46 \times 10^{-3}$, and $2.5 \times 10^{-2}$ for the first, central and the last particles, respectively, that is, part of Div $E_{t}$ on the particles' surfaces does not exceed $2.5 \%$ of the value of the $E$ components. In Fig. 9, the maximal values of $\operatorname{Div} E_{t}$ are presented. In fact, this value is two orders smaller, so the average value of $\operatorname{Div} E_{t}$ is practically one order smaller than presented ones. The numerical results, presented in Fig. 11, demonstrate that the value of Div $E_{t}$ depends on the distance $d$ between particles less, and this value becomes stable as $d$ grows. The results are related to central particle, $M=10^{3}, k=0.1$. For example, the value of $\operatorname{Div} E_{t}$ is equal to $2.8 \times 10^{-4}$ for $d=0.5$ and it stabilizes at the value $3.4 \times 10^{-4}$ at $d>1.2$ for $a=0.05$. Similar behavior of $\operatorname{Div} E_{t}$ is observed for $a=0.03$ and $a=0.01$. The maximal value of the ratio $\max \left(\operatorname{Div} E_{t}\right) / \min \left(E_{x, y, z}\right)$ is equal to $1.4 \%$ for the considered values of $a$ and $d$. The value of $\operatorname{Div} E_{t}$ slowly grows also as $d / a \leq 7$.

The dependence of $\operatorname{Div} E_{t}$ on the number $M$ of particles for various $a$ at the fixed value of $d$ is shown in Fig. 12. Here $k=0.1, d=0.5$. The maximal value of $\operatorname{Div} E_{t}$ is observed at $M=125$ and it is equal to $2.0 \times 10^{-3}, 1.15 \times 10^{-3}$, 


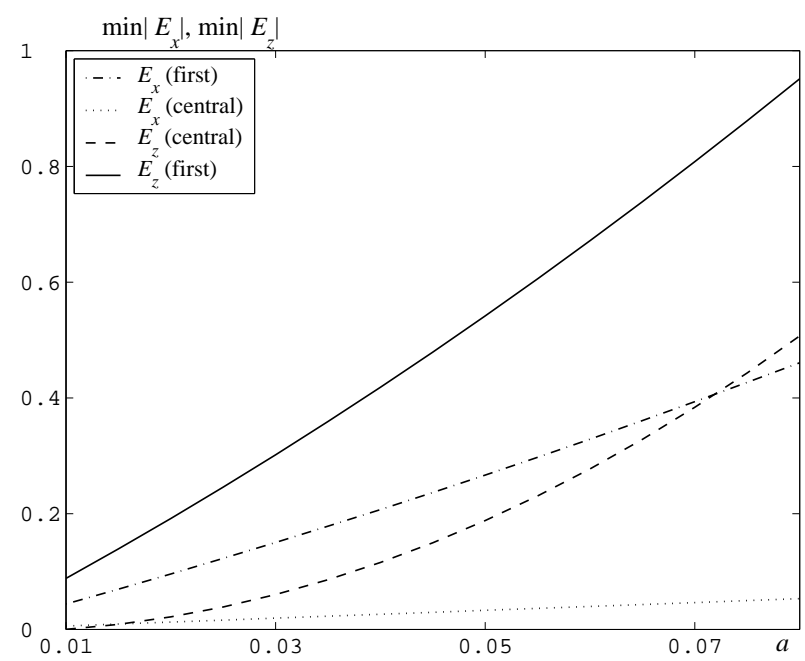

Figure 10: Minimal values of $E_{x}$ and $E_{z}$ components versus radius $a$ of particle

and $0.33 \times 10^{-3}$ for $a=0.05, a=0.03$, and $a=0.01$, respectively. This value becomes a local minimum for $M=9^{3}$, it grows slowly as $M$ increases and becomes stable at $M>15 \times 10^{3}$. The values of $\operatorname{Div} E_{t}$ at this $M$ are equal to $1.62 \times 10^{-3}, 0.73 \times 10^{-3}$, and $0.09 \times 10^{-3}$ for $a=0.05, a=0.03$, and $a=0.01$, respectively. The maximal value of the ratio $\max \left(\operatorname{Div} E_{t}\right) / \min \left(E_{x, y, z}\right)$ in the above calculations does not exceed $1.85 \%$.

Consequently, the obtained numerical results show that the condition $\operatorname{Div} E_{t}=$ 0 on the surfaces of the particles is satisfied with high accuracy.

\subsection{Justification of the Assumption $\operatorname{Div} \sigma_{m}=0$}

To check this assumption numerically we define the function $\sigma_{m}$ as the solution to integral equation of the second kind obtained in [21]

$$
\sigma_{m}(t)=A \sigma_{m}(t)+f_{m}(t)
$$

where $f_{m}=2\left[f_{e}(s), N_{s}\right]$ and

$$
\begin{gathered}
f_{e}(s)=\left[N_{s},\left[E_{e}(s), N_{s}\right]\right]-\frac{\zeta_{m}}{i \omega \mu_{0}}\left[\nabla \times E_{e}(s), N_{s}\right], \\
A \sigma_{m}=2 \zeta_{m} i \omega \varepsilon_{0}\left[N_{s},\left[N_{s}, \int_{S_{m}} g(s, t) \sigma_{m}(t)\right]\right] d t- \\
2 \int_{S_{m}}\left[N_{s},\left[\nabla_{s} g(s, t), \sigma_{m}(t)\right]\right] d t .
\end{gathered}
$$

This equation is solved by the collocation method [17]. The corresponding LAS has the form similar to (34)-(36). 


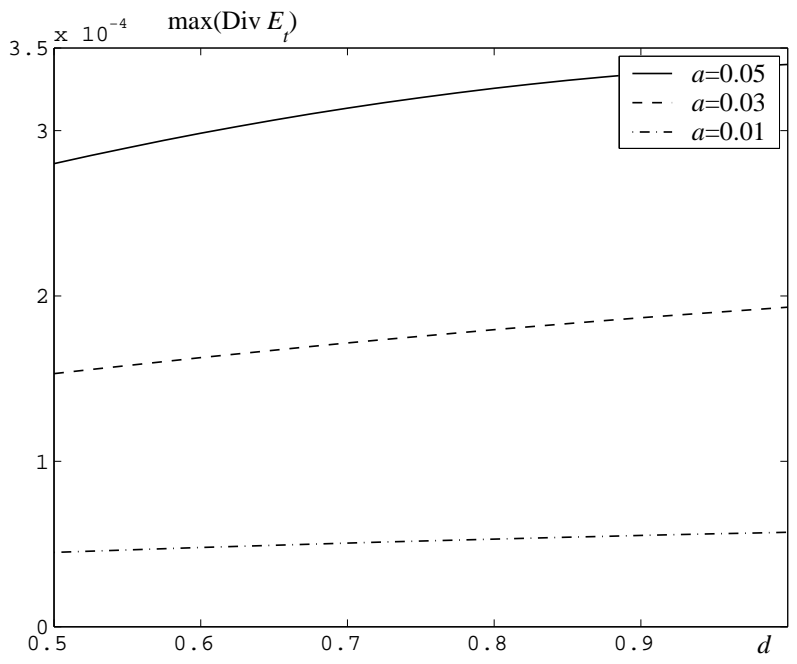

Figure 11: Maximal value of $\operatorname{Div} E_{t}$ versus distance $d$ between particles

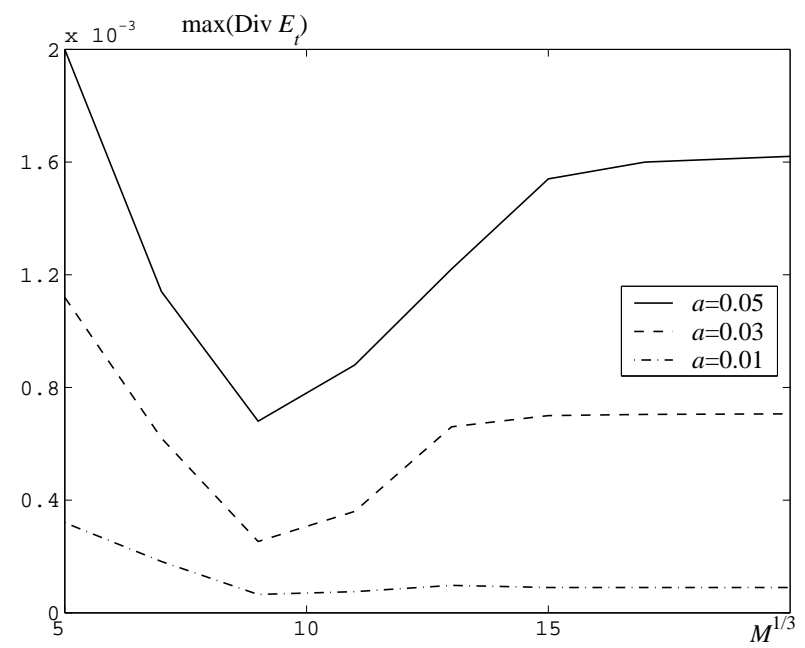

Figure 12: Maximal value of $\operatorname{Div} E_{t}$ versus number $M$ of particles 
First, we investigate the dependence of $\operatorname{Div} \sigma_{m}$ on the radius $a$ of particles at the fixed distance $d$ between them. The respective results are shown in Fig. 13. The maximal value of $\operatorname{Div} \sigma_{m}$ is shown for the first, central and last particles, total number of particles $M=10^{3}, k=0.1, d=0.5$. The maximal value of $\operatorname{Div} \sigma_{m}$ is given for the central particle at $a=0.01$ and it is equal to 0.0088 . This value is slightly smaller for the last and first particles (0.0055 and 0.0043, respectively). It decreases slowly as $a$ grows and becomes stable at $a>0.5$.

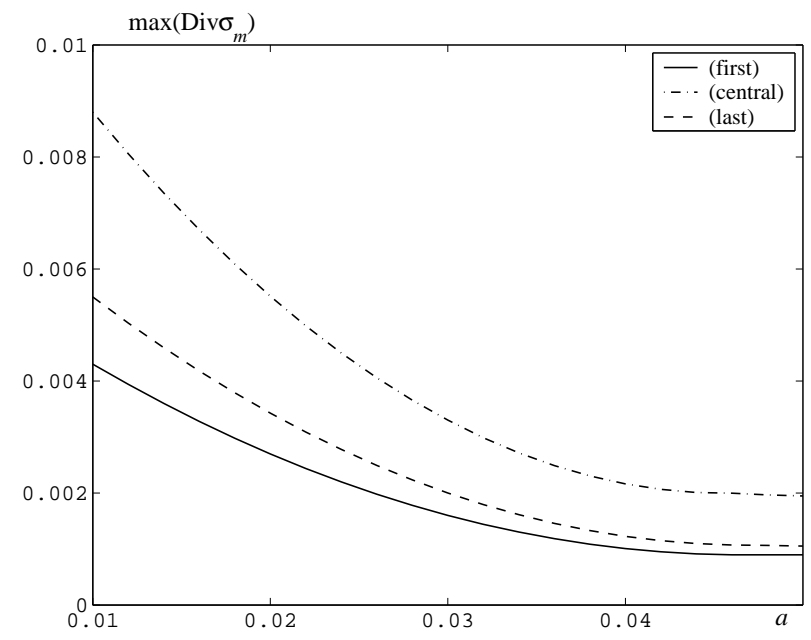

Figure 13: Maximal value of $\operatorname{Div} \sigma_{m}$ versus radius $a$ of particle

In Fig. 14, the $\theta$-th and $\varphi$-th components of $\sigma_{m}$ are presented. Use of the spherical components for calculation of $\operatorname{Div} \sigma_{m}$ is more convenient here. The $\sigma_{m \varphi}$ component is practically the same for the first, central and the last particles; therefore it is shown only for the central particle. One can see that the minimal value of $\sigma_{m \varphi}$ and $\sigma_{m \theta}$ components are two orders larger than the values of $\operatorname{Div} \sigma_{m}$. For example, the ratio $\max \left(\operatorname{Div} \sigma_{m}\right) / \min \left(\sigma_{m \theta}\right)$ is equal to $0.65 \%, 0.48 \%$, and $0.41 \%$ for the central, last and first particles, respectively, at $a=0.01$. This value for $\max \left(\operatorname{Div} \sigma_{m}\right) / \min \left(\sigma_{m \varphi}\right)$ equals to $2.42 \%, 1.51 \%$, and $1.18 \%$, that is the values of $\operatorname{Div} \sigma_{m}$ do not exceed several percents in comparison with the corresponding values of $\sigma_{m}$ components.

The dependence of the maximal value of $\operatorname{Div} \sigma_{m}$ on the distance $d$ between particles for several values of radius $a$ is shown in Fig. 15 for the central particle. The numerical results confirm that the values of the maximum of $\operatorname{Div} \sigma_{m}$ are at least two orders less than the values of $\sigma_{m \varphi}$ and $\sigma_{m \theta}$ components presented in Fig. 17. So, the ratio $\max \left(\operatorname{Div} \sigma_{m}\right) / \min \left(\sigma_{m \theta}\right)$ is equal to $0.41 \%, 0.38 \%$, and $0.32 \%$ for $a=0.01, a=0.03$, and $a=0.05$, respectively, at $d=0.5$. This value is equal to $0.18 \%, 0.18 \%$, and $0.17 \%$ for the above $a$ at $d=1.0$. One can see that this relation decreases slowly as $d$ increases. As for Div $E_{t}$, the quantity 


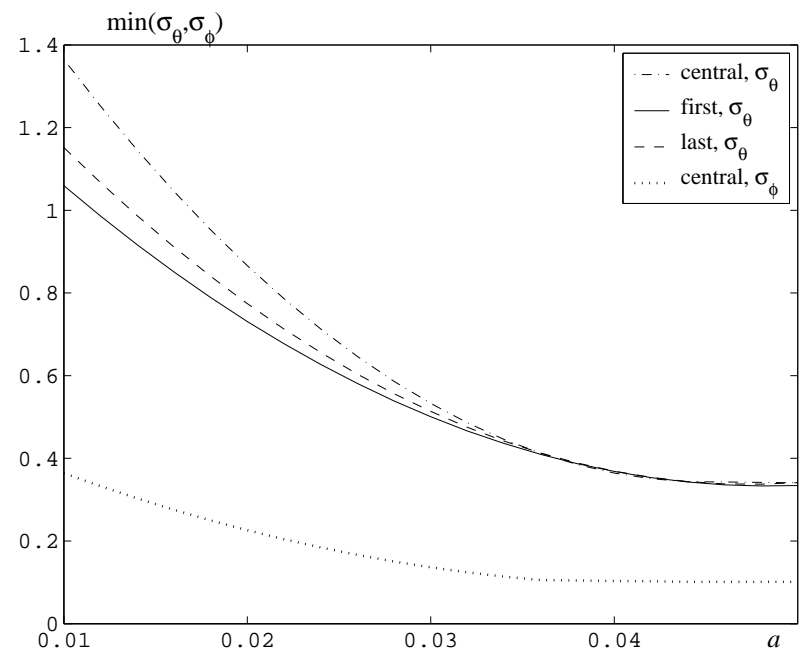

Figure 14: Minimal values of $\sigma_{m}$ components versus radius $a$ of particle

$\operatorname{Div} \sigma_{m}$ depends less on $d$ at fixed $a$ than on $a$ at fixed $d$.

The dependence of $\max \left(\operatorname{Div} \sigma_{m}\right)$ on the number $M$ of particles for several values of $a$ at fixed $d$ is shown in Fig. 17. Parameters $d=0.5, k=0.1$. The greatest value of $D i v \sigma_{m}$ is observed for $M=5^{3}$ at $a=0.01$ and it is equal to 0.0087 ; this value decreases as $M$ grows and it is equal to 0.0016 at $M=20^{3}$. This change is less for $a=0.03$ and $a=0.05$. The values of $\operatorname{Div} \sigma_{m}$ are 0.0033 and 0.0020 for $M=5^{3}$, and 0.0011 and 0.0006 for $M=20^{3}$. As example, the value of $\max \left(\operatorname{Div} \sigma_{m}\right) / \min \left(\sigma_{m \theta}\right)$ for $d=1.0$ is equal to $0.63 \%$ for $M=5^{3}$ and $0.21 \%$ for $M=20^{3}$ at $a=0.03$, and it is equal to $0.56 \%$ for $M=5^{3}$ and $0.17 \%$ for $M=20^{3}$ at $a=0.05$. These numerical results show that maximal value of the ratio $\max \left(\operatorname{Div} \sigma_{m}\right) / \min \left(\sigma_{m \theta}, \sigma_{m \varphi}\right)$ does not exceed a fraction of a percent. This confirms that the equality $\operatorname{Div} \sigma_{m}=0$ on the surface of particles can be used in numerical calculations.

\subsection{Creating Media with Piecewise-constant Distribution of Permeability}

The proposed approach allows one to create a media with a piecewise-constant magnetic permeability $\mu$. Such permeability can be realized either by embedding various numbers $M_{m}$ of particles into sub-domains $\Delta_{m}$ or by the variation of the function $h\left(x_{m}\right)$ in these sub-domains.

Both approaches have their advantages depending on the parameters $M, a$, and $d$ of $D$. In engineering it is often useful to have constant distribution of $\mu$ along certain direction (for example, along $z$ - and $y$-axis), and piecewiseconstant $\mu$ in the direction of $x$-axis.

In Fig. 18, such $\mu$ is formed by embedding various numbers of particles 


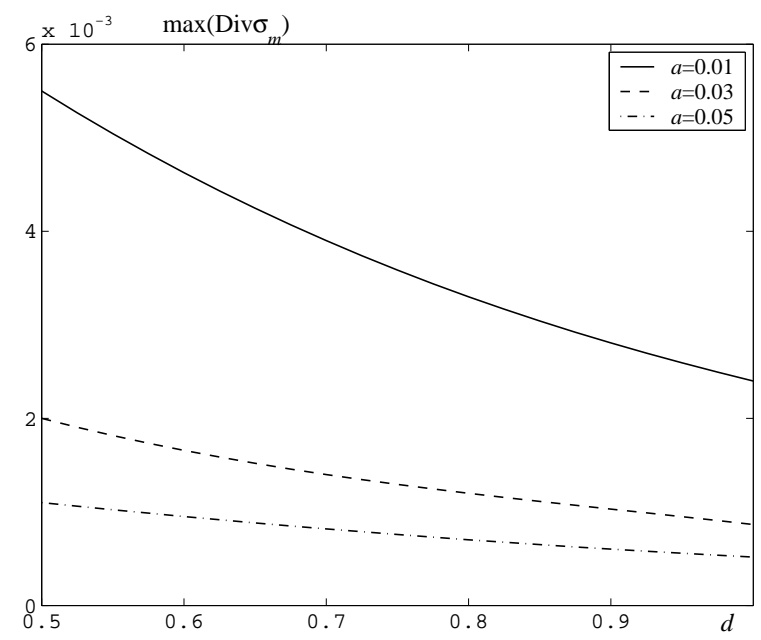

Figure 15: Maximal value of $\operatorname{Div} \sigma_{m}$ versus distance $d$ between particles

$M_{m}$ in sub-domains $\Delta_{m},(m=1,2,3): M_{1}=11 \times 11 \times 4, M_{2}=9 \times 9 \times 3$, $M_{3}=11 \times 11 \times 4$, respectively. At these values of $M_{m}$, the values of permeability are equal to 0.9229 in sub-domains $\Delta_{1}$ and $\Delta_{3}$, and the permeability is equal to 0.9410 in sub-domain $\Delta_{2}$. Such distribution of particles in the medium allows one to reach a difference in the $\mu$ values in the range of $2.2 \%$, the minimal deviation of $\mu$ from the $\mu_{0}$ is $5.9 \%$. In order to increase the difference $\mu_{m}$ for various $D_{m}$ it is necessary to increase the difference between $M_{m}$.

The distributions of $\mu$ obtained above allows one to form different distribution of $E_{x}$ and $E_{y}$ component of the total field. In Figs. 19 and 20, these components are shown in the far zone of $D\left(d_{f}=20 l_{D}\right)$, where $l_{D}$ is diameter of $D$, and $d_{f}$ is distance from center of $D$ to the far zone. The non-uniform distribution of $E_{x}-$ and $E_{y}$-components is explained by the different distribution of $\mu$ along $x-$ and $y$-axes.

Combining the ratio of values $M_{m}$, one can create the distribution of $\mu$ corresponding to various requirements. For example, distribution of $\mu$ which is shown in Fig. 21, is obtained at the following values of particles in sub-domains $\Delta_{m}, m=1,2,3: M_{1}=11 \times 11 \times 4, M_{2}=9 \times 9 \times 3, M_{3}=7 \times 7 \times 4$. At such values of $M_{m}$, the values of the permeability are equal to $0.8745,0.9026,0.9451$ in sub-domains $\Delta_{1}, \Delta_{2}$ and $\Delta_{3}$, respectively. The amplitudes $E_{x}$ and $E_{y}$ of EM field for this case have more complicated structure because of the larger change of $\mu$.

In Fig. 22, the piecewise-constant distribution of $\mu$, created by prescribing the various values of function $h\left(x_{m}\right)$ in four sub-domains $\Delta_{m},(m=1,2,3,4)$ of $D$, is shown. The number of particles in all sub-domains is equal to: $M=$ $11 \times 5 \times 5$, the values of $h\left(x_{m}\right)$ are the following $h_{1}=7 i, h_{2}=9 i, h_{3}=11 i$, $h_{4}=13 i$ (that is, $h\left(x_{m}\right)$ is piecewise-constant). The values of obtained $\mu$ and 


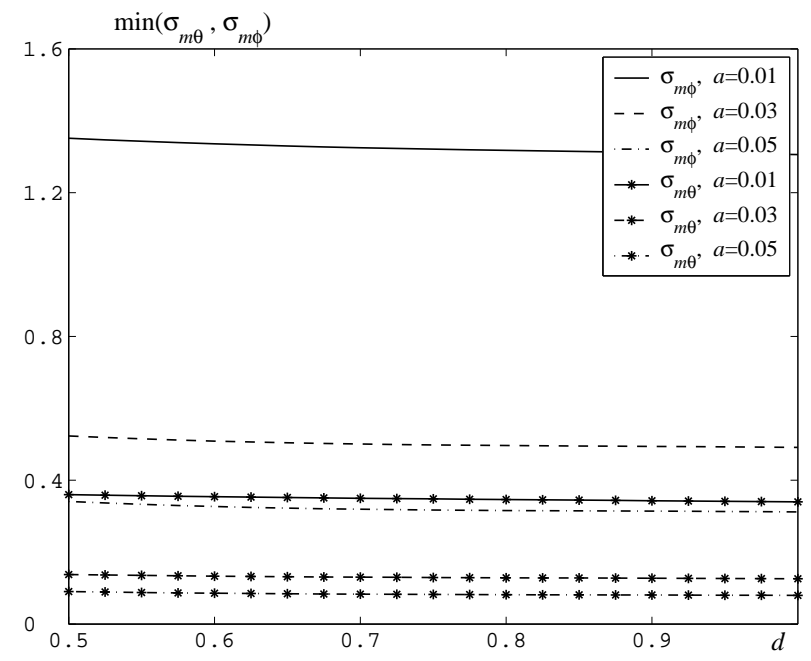

Figure 16: Minimal values of $\sigma_{m}$ components versus distance $d$ between particles

respective values of wave number $k$ are presented in Table 2. The minimal deviation of the obtained $\mu$ from the values $\mu_{0}$ is observed in sub-domain $\Delta_{1}$ and it is equal to $4 \%$, this deviation reaches $7 \%$ in sub-domain $\Delta_{4}$. In order to increase the deviation of $\mu$ in certain sub-domain $\Delta_{m}$ it is necessary to increase the value of $h_{m}$ in this sub-domain and keep the values of $h_{m}$ in the rest of sub-domains the same.

Table 2. The properties of resulting domain $D$ at piecewise-constant distribution of $h\left(x_{m}\right)$.

\begin{tabular}{llll}
\hline Subdomain & $h\left(x_{m}\right)$ & $\mu$ & $k$ \\
$\Delta_{1}$ & $-7 i$ & 0.9616 & 0.0981 \\
$\Delta_{2}$ & $-9 i$ & 0.9512 & 0.0976 \\
$\Delta_{3}$ & $-11 i$ & 0.9410 & 0.0970 \\
$\Delta_{4}$ & $-13 i$ & 0.9310 & 0.0965 \\
\hline
\end{tabular}

\section{Conclusions}

The numerical procedures for solving the EM wave scattering problem by one and many small impedance particles of an arbitrary shape are given. On this basis a method for creating the media with non-uniform distribution of magnetic permeability $\mu(x)$ is developed and tested numerically.

It is shown that the relative error of the asymptotic solution (17) compared with the Mie-type solution (52) in the paper [2] depends on the radius $a$ of the 


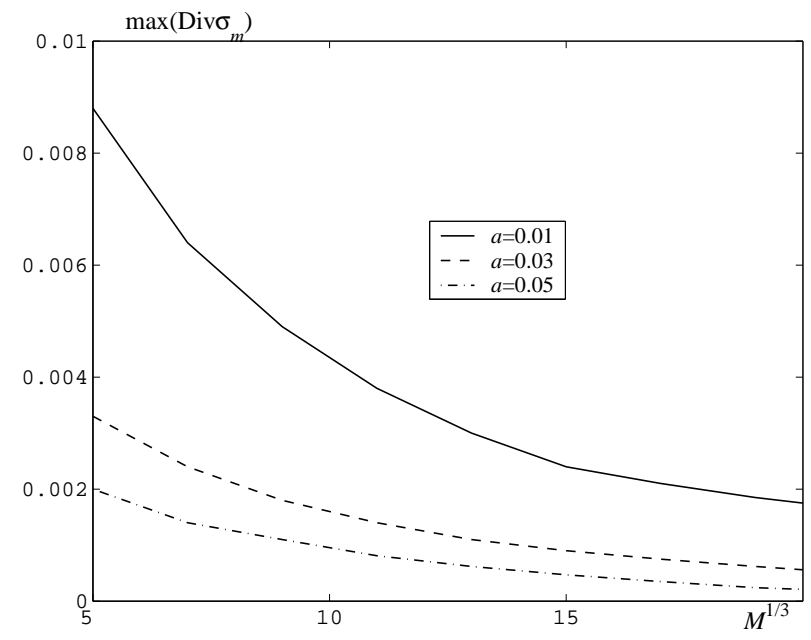

Figure 17: Maximal value of $\operatorname{Div} \sigma_{m}$ versus number $M$ of particles

particle, the distance $r$ to the observation point, and on the parameter $h$ which defines the surface impedance $\zeta$ of the particle.

The numerical results show that the assumptions $\operatorname{Div} E_{t}=0$ and $\operatorname{Div} \sigma_{m}=0$ on the surfaces of the particles are practically accurate: the relative error of these assumptions does not exceed $1.85 \%$ in the considered range of the parameters used for the solution of the scattering problem.

The computational results confirm the theoretical conclusion about the possibility to create media with piecewise-constant magnetic permeability $\mu$. Creating such a $\mu$ is achieved either by embedding various numbers $M_{m}$ of small particles in the sub-domains $\Delta_{m}$ of total domain $D$, or by changing the values $h\left(x_{m}\right)$ of the boundary impedances of the particles. Both approaches provide the possibility to change the initial values of $\mu$ in the range of $5 \%-40 \%$.

\section{References}

\section{References}

[1] Andriychuk M, Ramm A 2010 "Scattering by Many Small Particles and Creating Materials with a Desired Refraction Coefficient," Int. J. Computing Science and Mathematics 3 N 1/2 102-121

[2] Andriychuk M, Indratno S, Ramm A 2012 "Electromagnetic Wave Scattering by a Small Impedance Particle: Theory and Modeling,' Optics Communications 285 1684-1691 


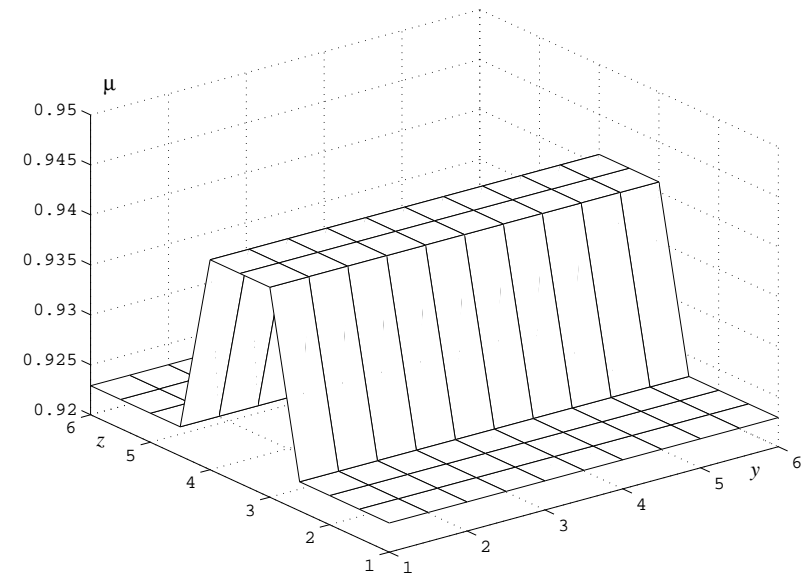

Figure 18: The piecewise-constant distribution of $\mu$ corresponding to two various $M_{m}$

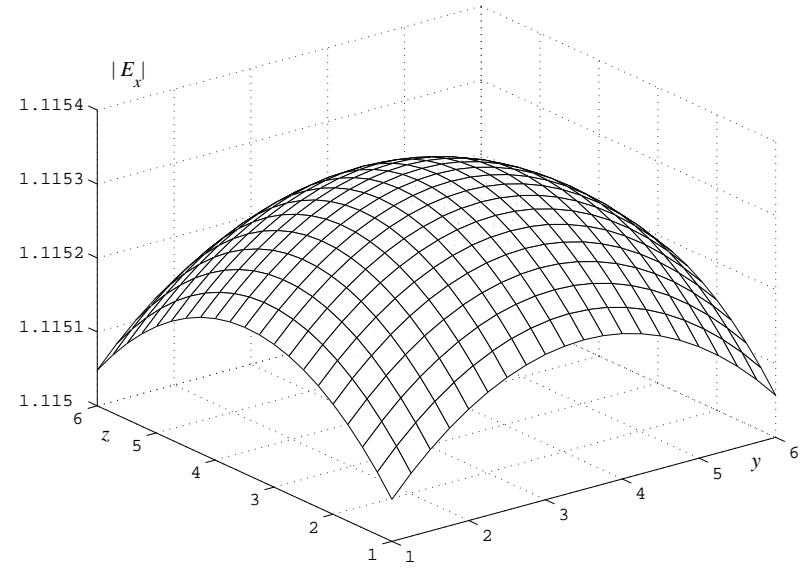

Figure 19: $E_{x}$ component of field in the far zone 


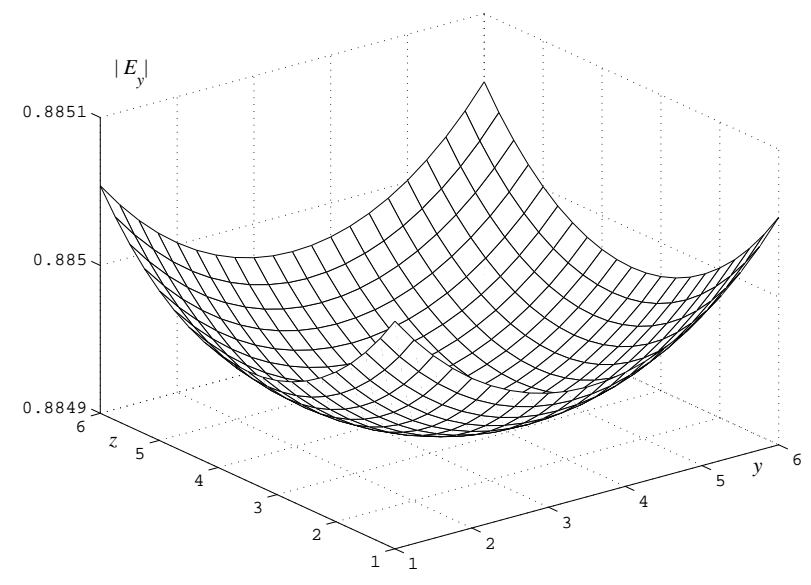

Figure 20: $E_{y}$ component of field in the far zone

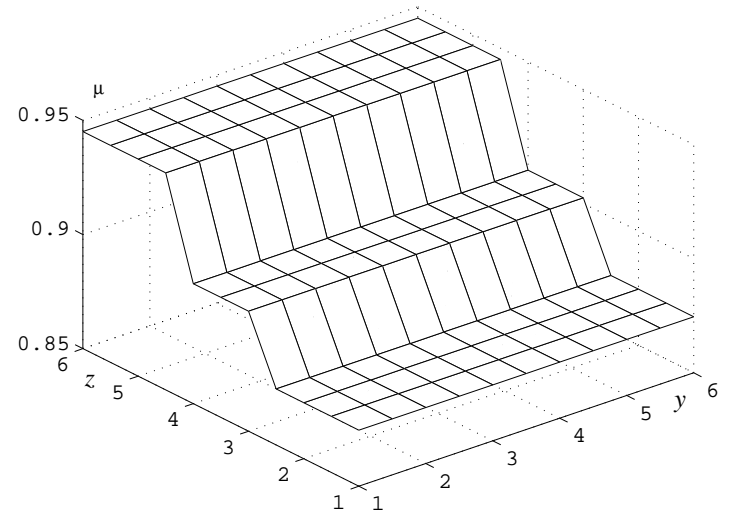

Figure 21: The piecewise-constant distribution of $\mu$ corresponding to three various $M_{m}$ 


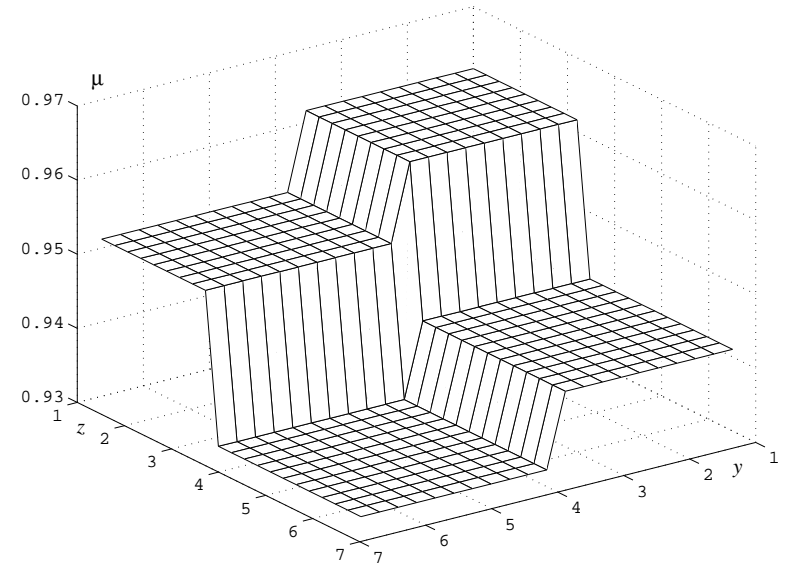

Figure 22: The piecewise-constant distribution of $\mu$ corresponding to four various values of $h\left(x_{m}\right)$

[3] Cioranescu D, Donate P 1999 An Introduction to Homogenization (New York: Oxford Univ. Press)

[4] Hoang N, Ramm A 2009 "Dynamical Systems Method for Solving Finiterank Operator Equations," Ann. Polon. Math. 95 N 1 77-93

[5] Jikov V.V., Kozlov S.M., Oleinik O.A. (1994) Homogenization of Differential Operators and Integral Functionals (Berlin: Springer-Verlag)

[6] Kantorovich L, Akilov G 1980 Functional Analysis (New York: Pergamon Press)

[7] Korn G, Korn T 1968 Mathematical Handbook for Scientists and Engineers (New York: McGraw-Hill Book Company)

[8] Krasnosel'skii M et al 1972 Approximate Solutions of Operator Equations ( Groningen: Wolters-Noordhoff)

[9] Landau L, Lifshitz E 1982 Electrodynamics of Continuous Media ( London: Pergamon Press)

[10] Marchenko V, Khruslov E 2006 Homogenization of Partial Differential Equations (Boston: Birkhauser)

[11] Marqués R, Martel J, Mesa F, Medina F 2002 "Left-Handed-Media Simulation and Transmission of EM Waves in Subwavelength Split-RingResonator-Loaded Metallic Waveguides," Physical Review Letters 89 (18) 183901-1-183901-4 
[12] Müller C 1969 Foundations of the Mathematical Theory of Electromagnetic Waves (Berlin: Springer-Verlag)

[13] Ramm A 1986 , Scattering by Obstacles (Dordrecht: D. Reidel)

[14] Ramm A 2007 "Distribution of Particles which Produces a "Smart" Material, Journ. Stat. Phys. 127 N 5 915-934

[15] Ramm A 2007 "Many-body wave scattering by small bodies and applications," J. Math. Phys. 48 N 10

[16] Ramm A 2008 "Wave Scattering by Many Small Particles Embedded in a Medium," Phys. Lett. A 372/17 3064-3070

[17] Ramm A 2009 "A Collocation Method for Solving Integral Equations," Intern. Journ. of Comput. Sci. and Mathem. 3 N 2 222-228

[18] Ramm A 2010 "Electromagnetic wave scattering by many small particles and creating materials with a desired permeability," Progress in Electromagnetic Research, M (PIER M) 14 193-206

[19] Ramm A 2013 "Many-body Wave Scattering Problems in the Case of Small Scatterers," J. of Appl. Math. and Comput., (JAMC) 41 N $1473-500$

[20] Ramm A 2011 "Electromagnetic Wave Scattering by a Small Impedance Particle of Arbitrary Shape," Optics Communications 284 3872-3877.

[21] Ramm A 2013 "Electromagnetic Wave Scattering by Small Impedance Particles of an Arbitrary Shape," Journ of Appl. Math. and Computing (JAMC) $43 \mathrm{~N}$ 1, (2013), 427-444.

DOI: $10.1007 / \mathrm{s} 12190-013-0671-3$

[22] Ramm A 2013 Scattering of Acoustic and Electromagnetic Waves by Small Bodies of Arbitrary Shapes. Applications to Creating New Engineered Materials, (New York: Momentum Press)

[23] Smith D, Padilla W, Vier D, Nemat-Nasser S and Schultz S 2000 "Composite Medium with Simultaneously Negative Permeability and Permittivity," Physical Review Letters 84 (18) 4184-4187

[24] Wu B.-I, Wang W, Pacheco J, Chen X, Grzegorczyk T and Kong J 2005 "A Study of Using Metamaterials as Antenna Substrate to Enhance Gain," Progress in Electromagnetics Research 51 295-328 